\title{
Calibrating Photometric Stereo by Holistic Reflectance Symmetry Analysis
}

\author{
Zhe Wu Ping Tan \\ National University of Singapore
}

\begin{abstract}
Under unknown directional lighting, the uncalibrated Lambertian photometric stereo algorithm recovers the shape of a smooth surface up to the generalized bas-relief $(G B R)$ ambiguity. We resolve this ambiguity from the halfvector symmetry, which is observed in many isotropic materials. Under this symmetry, a 2D BRDF slice with low-rank structure can be obtained from an image, if the surface normals and light directions are correctly recovered. In general, this structure is destroyed by the GBR ambiguity. As a result, we can resolve the ambiguity by restoring this structure. We develop a simple algorithm of auto-calibration from separable homogeneous specular reflection of real images. Compared with previous methods, this method takes a holistic approach to exploiting reflectance symmetry and produces superior results.
\end{abstract}

\section{Introduction}

Photometric stereo is an important research topic in computer vision. It provides direct access to surface normal$\mathrm{s}$, which not only are crucial in photo-realistic rendering, but also help improve 3D reconstruction accuracy [12, 13]. Auto-calibration algorithms are practically important for photometric stereo, since conventional algorithms (e.g. [25]) requiring known illumination conditions involve additional efforts of calibration. Under unknown directional lighting, it is well known that surface normals of a Lambertian object can only be determined up to a linear ambiguity [10]. Later it is shown $[26,3]$ that this ambiguity can be reduced to the generalized bas-relief (GBR) ambiguity by enforcing the integrability constraint. This paper focuses on further resolving the GBR ambiguity.

Reflectance of an opaque material is described by the bidirectional reflectance distribution function (BRDF), which is a function of the incoming and outgoing light directions in a local coordinate system. Reflectance of many real-world objects satisfies various symmetries, e.g. isotropy and reciprocity, which provides additional information to resolve the GBR ambiguity. As demonstrated in [20, 22], the GBR ambiguity can be solved using 'isotropic pairs' and 'reciprocal pairs' identified from one

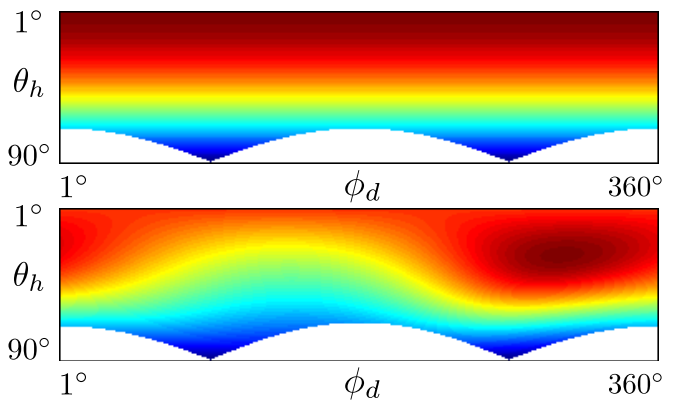

Figure 1. Synthesized 2D slices of a bivariate BRDF. Values are color-coded: red means larger BRDF values, blue indicates smaller values, and white regions are undefined for a BRDF. The horizontal and vertical axes are two angles $\phi_{d}$ and $\theta_{h}$, which are defined in Section 3.1. The upper slice, which is obtained from ground truth normals and light direction, is constant along each row, while clearly this structure does not hold for the bottom slice estimated using GBR-distorted normals and light direction.

or two images. Surface points with specular spike [7, 6] or diffuse maxima [8] can also resolve the GBR ambiguity. However, these methods all require carefully identified special surface points, which are easily affected by image noise.

We solve the GBR ambiguity by a holistic analysis of half-vector symmetry, which suggests the BRDF value stays unchanged when rotating the incoming and outgoing light directions as a fixed pair around their bisector. This symmetry is closely related to the barycentric parameterization of isotropic BRDFs [19], and can be elegantly expressed in the halfway/difference parameterization [15]. Given the correct surface normals and light directions, we can obtain a 2D BRDF slice from each image of a curved isotropic surface. If the BRDF is half-vector symmetric, this $2 \mathrm{D}$ slice should form a special low-rank matrix when it is properly parameterized, as illustrated at the top of Figure 1. However, as we have observed and will present in subsequent sections, such a structure is generally destroyed when normals and light directions are distorted by a GBR transformation, as shown at the bottom of Figure 1. Restoring the special structure of 2D BRDF slices can resolve the GBR ambiguity. We propose a simple algorithm to find the solution based on this observation. 
The contribution of this paper mainly lies in: 1) proposing half-vector symmetry as a novel cue to solve the GBR ambiguity; 2) proving that half-vector symmetry resolves the GBR ambiguity; 3) providing a simple auto-calibration algorithm based on a holistic analysis of this symmetry.

\subsection{Related Work}

Woodham [25] proposed the first photometric stereo method with directional lighting information known a priori. Hayakawa [10] proved that under unknown directional illumination, normals of a Lambertian surface can only be recovered up to a linear transformation. This ambiguity is reduced to the GBR ambiguity by enforcing the integrability constraint $[26,3]$.

Different methods have been proposed to resolve the GBR ambiguity. Besides those utilizing interreflection [5] or special lighting configurations [28], the other methods can be divided into two categories. The first category of methods resolves the ambiguity by analyzing reflectance properties. Earlier methods rely on specific reflectance models. Georghiades [9] adopted the Torrance-Sparrow model [24] to tackle the problem. In [7, 6], the authors assumed specular-spike reflectance and showed that the ambiguity is solved by detected specular spots in images. More recen$\mathrm{t}$ methods exploit general reflectance symmetries. Tan et al. [20, 22, 21] exploited isotropy and reciprocity to recover GBR parameters from carefully identified 'isotropic pairs' and 'reciprocal pairs' in a single image.

In the second category, priors on surface albedos are exploited. Alldrin et al. [1] recovered the GBR parameters by assuming the true distribution of surface albedo has small entropy. Shi et al. [17] identified surface points with the same albedo but different normals to resolve the ambiguity. In a recent work [8], the authors assumed smoothly varying surface albedos in order to locate the 'lambertian diffuse maxima', which are then used in a robust estimation framework to estimate the GBR paramters.

\section{Background}

Assume a Lambertian object is illuminated by distant light sources and imaged by a fixed orthographic camera. If interreflection and shadow are ignored, the intensity $i_{p f}$ of a pixel $p$ under the light source $s_{f}$ is given by

$$
i_{p f}=\rho_{p} \mathbf{n}_{p}^{\top} s_{f},
$$

where $\rho_{p}$ and $\mathbf{n}_{p}$ are the albedo and normal of the pixel $p$ respectively, the norm $\left\|s_{f}\right\|$ and normalized vector $\mathbf{s}_{f}$ indicate the light intensity and direction. Note that we use bold lowercase letters to indicate normalized vectors. For all $P$ pixels in an image under $F$ different light directions, Equation 2.1 can be written in a matrix form

$$
I=N^{\top} S \text {. }
$$

Each column of $I$ is an image under one of the light sources and each row is the intensity profile of a pixel under al1 different illumination conditions. Columns of $N$ are surface normals multiplied by their corresponding albedos, and columns of $S$ are light directions scaled by their intensities. For a smooth surface, normals and light directions can be recovered to an unknown GBR transformation $G$ [3]:

$$
I=N^{\top} S=(G N)^{\top}\left(G^{-\top} S\right)=\hat{N}^{\top} \hat{S},
$$

where $\hat{N}=G N, \hat{S}=G^{-\top} S$ and $G$ has the following matrix form

$$
\mathbf{G}=\left(\begin{array}{lll}
\lambda & 0 & \mu \\
0 & \lambda & \nu \\
0 & 0 & 1
\end{array}\right) .
$$

As is shown in Equation 2.3, a normal $\mathbf{n}$ and a light direction $\mathbf{s}$ are distorted in the following way

$$
\hat{\mathbf{n}}=\frac{G \mathbf{n}}{\|G \mathbf{n}\|}, \hat{\mathbf{s}}=\frac{G^{-\top} \mathbf{s}}{\left\|G^{-\top} \mathbf{s}\right\|} .
$$

The aim of auto-calibration is to restore every $\mathbf{n}$ and each $\mathbf{s}$ from $\hat{\mathbf{n}}$ and $\hat{\mathbf{s}}$ respectively by recovering $\lambda, \mu$ and $\nu$.

\section{Half-Vector Symmetry and GBR}

In this section, we will introduce half-vector symmetry of BRDFs and present the special low-rank matrix structure enforced by it. After that, we will examine how the GBR ambiguity destroys the structure of this matrix.

\subsection{Half-Vector Symmetry}

BRDF is a function of incoming and outgoing light directions $\left(\boldsymbol{\omega}_{\text {in }}, \boldsymbol{\omega}_{\text {out }}\right)$ in a local coordinate system. Rusinkiewitz [15] proposed to use four spherical coordinates $\left(\theta_{h}, \phi_{h}, \theta_{d}, \phi_{d}\right)$ to parameterize a BRDF as

$$
f\left(\boldsymbol{\omega}_{\text {in }}, \boldsymbol{\omega}_{\text {out }}\right)=f\left(\theta_{h}, \phi_{h}, \theta_{d}, \phi_{d}\right) .
$$

Angles in Equation 3.1 are illustrated in Figure 2 and explained here. First of all, the half vector is defined as the bisector of lighting and viewing directions, i.e. $\mathbf{h}=$ $\frac{\boldsymbol{\omega}_{\text {in }}+\boldsymbol{\omega}_{\text {out }}}{\left\|\boldsymbol{\omega}_{\text {in }}+\boldsymbol{\omega}_{\text {out }}\right\|}$. In a local coordinate system where the surface normal is aligned with the z-axis, $\theta_{h}$ and $\phi_{h}$ are the azimuthal and polar angles of $\mathbf{h}$ respectively. $\theta_{h}$ is called half angle. $\theta_{d}$ is named difference angle and is defined as the angle between $\mathbf{h}$ and $\boldsymbol{\omega}_{i n} . \phi_{d}$ indicates the rotation angle of $\boldsymbol{\omega}_{\text {in }}$ and $\boldsymbol{\omega}_{\text {out }}$ as a pair around the half vector $\mathbf{h}$.

Common BRDF symmetries can be elegantly expressed using this parameterization. One of the various symmetries widely observed in real-world materials is isotropy, which means BRDF values stay unchanged as the lighting and viewing directions are rotated as a fixed pair around the normal. Thus, isotropy reduces the BRDF to a $3 \mathrm{D}$ function $f\left(\theta_{h}, \theta_{d}, \phi_{d}\right)$. Many isotropic materials also satisfy the half-vector symmetry, which suggests that BRDF values are invariant with rotation of lighting and viewing direction$s$ around the half vector. In this case, the BRDF does not 


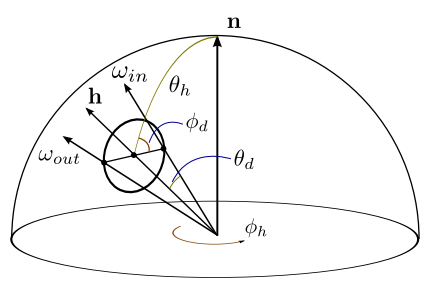

Figure 2. Halfway/difference parameterization of BRDF [15]

depend on $\phi_{d}$ and is further reduced to a bivariate function $f\left(\theta_{h}, \theta_{d}\right)$.

This kind of bivariate BRDF model is reported in previous works. For example, Stark et al. [19] studied several traditional parametric reflectance models and showed that they are bivariate. Shi et al. [18] further used a biquadratic function to represent bivariate BRDFs. Romeiro et al. [14] evaluated the validity of such a representation on the MERL BRDF database [11] and concluded that it can be used to represent most materials in the database to high accuracy. Besides, the same bivariate BRDF representation has already been adopted in calibrated photometric stereo [2] and reflectometry [14].

\subsection{Structured 2D BRDF Slice}

The pixel intensity of a general isotropic surface is calculated as $I=f\left(\theta_{h}, \theta_{d}, \phi_{h}\right)(\mathbf{n} \cdot \mathbf{s})$. We ignore the light intensity $\|s\|$ here for notation simplicity. Under the assumption of directional lighting, orthographic camera and homogeneous surface reflectance, pixel intensities in an image are determined as

$$
I=f_{\theta_{d}}\left(\theta_{h}, \phi_{d}\right)(\mathbf{n} \cdot \mathbf{s}),
$$

where $f_{\theta_{d}}\left(\theta_{h}, \phi_{d}\right)=f\left(\theta_{h}, \theta_{d}, \phi_{d}\right)$ is a 2D slice of the original BRDF.

For a curved surface with abundant normals, e.g. a sphere, when both the normals $\mathbf{n}$ and light direction $\mathbf{s}$ are known, we can estimate a 2D slice of the BRDF, namely $f_{\theta_{d}}\left(\theta_{h}, \phi_{d}\right)$, based on Equation 3.2. This BRDF slice can be arranged into a matrix form in the range $\theta_{h} \in\left[0, \frac{\pi}{2}\right]$, $\phi_{d} \in[0,2 \pi]$. For a bivariate BRDF, $f$ does not depend on $\phi_{d}$. Thus each row of the matrix is constant. Such a lowrank structure can be clearly seen at the top of Figure 1.

\subsection{GBR-Distorted 2D BRDF Slice}

When surface normals and light direction are distorted by a GBR transformation as in Equation 2.5, the 2D BRDF slice estimated from Equation 3.2 no longer has the lowrank structure. An example from synthetic data is shown at the bottom of Figure 1. This observation motivates us to resolve the GBR ambiguity by restoring the low-rank structure of BRDF slices.

In the special case that the lighting and viewing directions coincide, the low-rank property is preserved by the classic bas-relief ambiguity, i.e. $\mu=\nu=0$ in Equation 2.4.

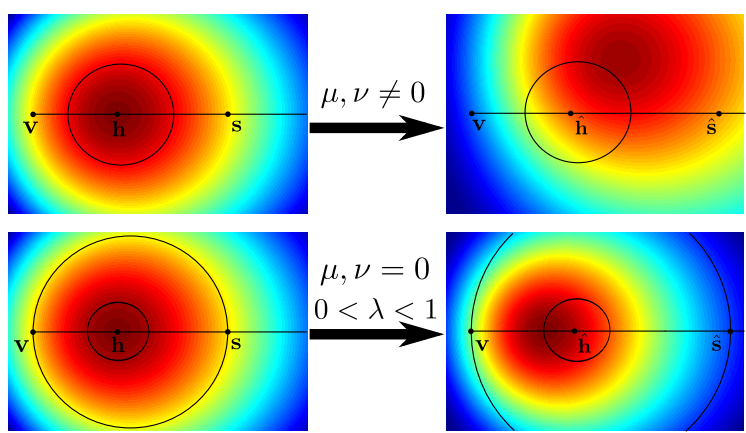

Figure 3. Projective plane with color-coded BRDF values. A black ellipse corresponds to a row in the BRDF slice of Figure 1. Top row shows the effect of a GBR transformation $(\mu, \nu \neq 0)$; bottom row shows the case of a classic bas-relief transformation with $0<$ $\lambda<1$. Before GBR transformation, the values on an ellipse are the same, while this is not true after GBR transformation.

In more general cases, however, a GBR transformation will destroy the low-rank structures of 2D BRDF slices. In fac$\mathrm{t}$, for a general bivariate $\mathrm{BRDF}$, we are able to prove the following proposition.

Proposition 1. Any GBR transformation cannot simultaneously perserve the special low-rank structure of bivariate BRDF slices estimated from two images whose light directions are not coplanar with the viewing direction.

Here we give some intuitive explanations with illustrative figures. Please refer to appendix for a formal proof.

We consider the problem on the projective plane where a $3 \mathrm{D}$ unit vector $(x, y, z)$ is represented by a point $(x / z, y / z)$. Viewing direction $\mathbf{v}=(0,0,1)^{\top}$, light direction $\mathbf{s}$, half vector $\mathbf{h}$ and every surface normal $\mathbf{n}$ can find their corresponding points on this plane. From an image of a curved surface, e.g. a sphere, we can observe sufficient number of points (surface normals) of this plane. Given the lighting and surface normal directions, a BRDF value can be estimated at each pixel of an image. By encoding these BRDF values into colors and mapping them to the projective plane according to the normal at each pixel, we obtain a 'BRDF map' shown in Figure 3, where red indicates larger values.

First of all, as marked by the black ellipse in Figure 3, points with the same $\theta_{h}$ form an ellipse around $\mathbf{h}$, with one of its symmetry axis being the line $v s$ connecting $\mathbf{v}$ and $\mathbf{s}$. For a bivariate BRDF, the BRDF value should be constant along each ellipse, since $\theta_{h}$ is fixed for those points on the same ellipse and $\theta_{d}$ is fixed for all pixels in the same image. These ellipses correspond to rows in the matrix representation of the 2D BRDF slice in Figure 1.

Now we show how a GBR transformation can change the structure. The GBR transformation moves normals and the light direction in different ways, as shown in Equation 2.5. In fact, the transformed light direction $\hat{\mathbf{s}}$ will still lie on the line $v s$, according to the equation 


$$
\hat{\mathbf{s}}=\frac{G^{-T} \mathbf{s}}{\left\|G^{-T} \mathbf{s}\right\|} \simeq\left(\begin{array}{c}
s_{x} \\
s_{y} \\
-\mu s_{x}-\nu s_{y}+\lambda s_{z}
\end{array}\right)
$$

where the symbol $\simeq$ means equal up to a scale. So the transformed half vector $\hat{\mathbf{h}}$ will also stay on the line vs. On the other hand, a GBR transformation will translate all normals (along with the BRDF map associated them) by a displacement $(\mu, \nu)$ and scale them by $\lambda$. Besides, BRDF values are also changed since they are estimated from pixel intensities and the GBR transformed shading $\hat{\mathbf{n}}^{\top} \hat{\mathbf{s}}$ using Equation 3.2.

In general, the different motions of normals and the light direction will make the BRDF value change along the transformed ellipse(consists of points forming the same half angle with $\hat{\mathbf{h}}$ ). As a result, the low-rank structure of the 2D BRDF slice in Figure 1 will be destroyed. For example, the top of Figure 3 shows the case of non-zero $\mu, \nu$. The transformed BRDF map has varying values along the ellipse around $\hat{\mathbf{h}}$. Similarly, at the bottom of Figure 3, we consider the case of $\mu=\nu=0$ and $\lambda \neq 1$, i.e. the classic bas-relief ambiguity. $\lambda<1$ is assumed here. In this case, the GBR transformation moves the light direction $\mathbf{s}=\left[s_{x}, s_{y}, s_{z}\right]^{\top}$ to $\hat{\mathbf{s}}$, which is even further from $\mathbf{v}$ (the origin). This can be seen from the following equation

$$
\hat{\mathbf{s}}=\left(\begin{array}{ccc}
\frac{1}{\lambda} & 0 & 0 \\
0 & \frac{1}{\lambda} & \\
0 & 0 & 1
\end{array}\right)\left(\begin{array}{c}
s_{x} \\
s_{y} \\
s_{z}
\end{array}\right) \simeq\left(\begin{array}{c}
s_{x} /\left(\lambda s_{z}\right) \\
s_{y} /\left(\lambda s_{z}\right) \\
1
\end{array}\right) .
$$

At the same time, normals and the associated BRDF map will be scaled and shrinked toward the origin $\mathbf{v}$. So the transformed BRDF value is no longer constant along the transformed ellipse. This again breaks the low-rank structure of the 2D BRDF slice.

\section{Auto-Calibrating Photometric Stereo}

We follow the dichromatic reflectance model [16] and assume that the reflectance of an object is the sum of a diffuse component and a homogeneous specular componen$\mathrm{t}: f\left(\omega_{\text {in }}, \omega_{\text {out }}, x\right)=\rho(x)+f_{s}\left(\omega_{\text {in }}, \omega_{\text {out }}\right)$, where $x$ indicates a surface point, $\rho$ is the diffuse albedo and $f_{s}$ is the specular BRDF. Given multiple images taken under varying lightings and a fixed viewpoint, it is relatively easy to separate the diffuse and specular components using existing techniques [16, 23]. From the diffuse images, we are able to recover surface normals and light directions up to a GBR transformation by the uncalibrated photometric stereo method [26].

By assuming the specular BRDF is bivariate, it is guaranteed by our earlier discussion that surface normals are correctly recovered iff the low-rank structure in estimated specular BRDF slices is restored.

One natural idea of restoring the low-rank structure is to use the established TILT technique [27], which recovers a low-rank pattern via domain transformation. In our case, however, both the position of each point (corresponding to a normal) on the 2D BRDF slice and its associated BRDF value are changed by a GBR transformation. Thus, TILT is not suitable for our problem and we need to resort to other solutions. We formulate a simple optimization algorithm to estimate the GBR parameters given normals and light directions up to a GBR ambiguity, together with a set of specular images.

We first define an objective function to measure how well the estimated 2D BRDF slice satisfies the special 'low-rank' constraint, which in our case means each row is constant. The most straightforward measure is defined as the sum of variances along each row(the axis of $\phi_{d}$ ). It is obvious that the correct GBR parameters should correspond to the unique global minimum of the objective function.

However, there are some practical issues in adopting such a measure. Firstly, with a real image of limited resolution, some entries of the 2D BRDF slice are missing because the corresponding normals are not observed in the image. Different rows of the matrix have different number$\mathrm{s}$ of observations. Thus we give higher weights to rows of a larger number of valid observations, since the variances calculated from those rows are more reliable. Secondly, the absolute variance level is biased by BRDF values. In other words, rows with larger BRDF values tend to have larger variances. So we divide the variance of each row by the square of its mean value for normalization. Thirdly, the BRDF values estimated from Equation 3.2 at large $\theta_{h}$ are usually noisy because the shading term $\mathbf{n}^{\top} \mathbf{s}$ tends to be small over those regions. So we only use the top 20 rows $\left(\theta_{h}=1^{\circ}, 2^{\circ}, \ldots, 20^{\circ}\right)$ when evaluating the variance. Here is the objective function we used,

$$
\min _{G} \sum_{i=0}^{N-1} \sum_{\theta_{h}=1}^{20} \frac{k_{i, \theta_{h}}}{\sum_{\theta=1}^{20} k_{i, \theta}} \frac{\operatorname{Var}\left[f_{i}\left(\theta_{h}, \phi_{d}\right)\right]}{\operatorname{Mean}^{2}\left[f_{i}\left(\theta_{h}, \phi_{d}\right)\right]} .
$$

In this function, $N$ is the number of input specular images and we can estimate a 2D BRDF slice $f_{i}$ from each image. The integer $k_{i, \theta_{h}}$ is the number of valid observations in row $\theta_{h}$ of slice $i$. Note that the calculation of variance and mean are evaluated only on valid entries.

Empirically, we found it useful to add one more constraint to the objective function 4.1. We require the sum of intensities (BRDF value times $\mathbf{n}^{\top} \mathbf{s}$ ) in the top 20 rows makes up at least $5 \%$ of that of the whole $2 \mathrm{D}$ slice. If this condition is not satisfied in a BRDF slice, a large constant penalty value will replace the sum of variance for that slice. This simple practice helps exclude some degenerated solutions, e.g. a very small $\lambda$ compressing the whole BRDF map to a single point.

The proposed objective function is difficult to optimize because both the position and value of the BRDF map depend on the GBR transformation. We simply adopt a 


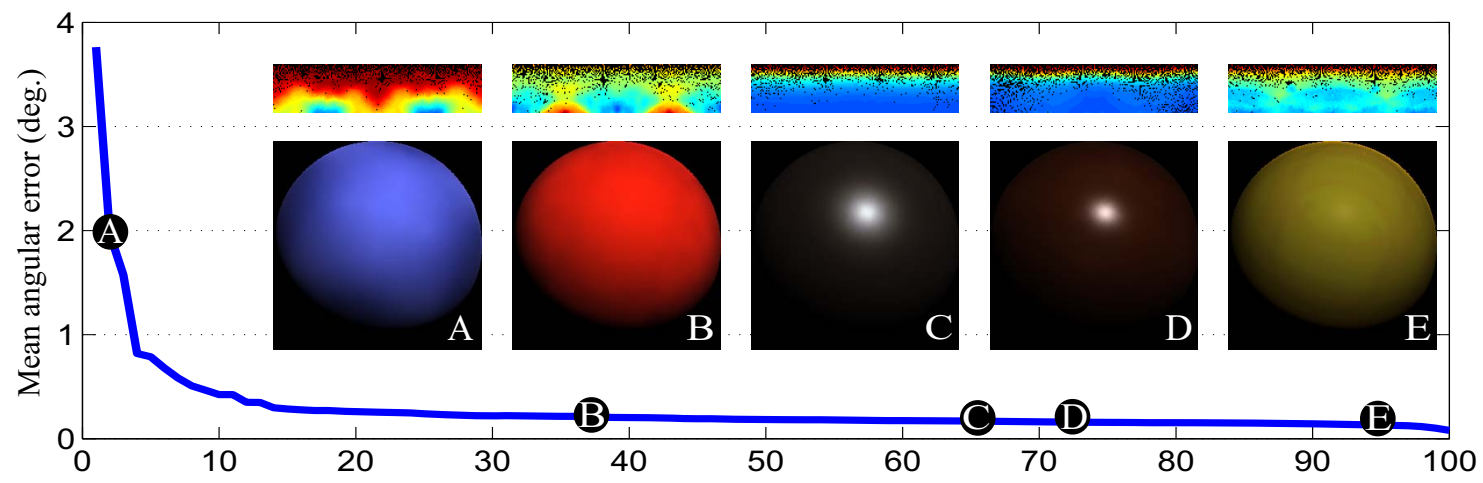

Figure 4. Error plot of results on MERL database. BRDFs are sorted according to error values. Five materials from the database are presented using a rendered sphere. One of the recovered BRDF slice(only the range $\theta_{h} \in\left[0^{\circ}, 40^{\circ}\right]$ is shown) is also shown above the rendered sphere.

coarse-to-fine search for optimization. The objective function is first evaluated at coarsely sampled grid points in the parameter space. We then search nearby the optimal grid point with higher sampling rate. Empirically, we find the objective function is smooth and this multi-resolution search can generate good results. As in [1], the search space is restricted to $-5 \leq \mu, \nu \leq 5,0<\lambda \leq 5$. The initial sampling step is set as 0.5 . After each iteration, both search range and sampling step are reduced to $\frac{1}{5}$ of the coarser one. Three iterations suffices to find an accurate estimation of GBR parameters.

\section{Experimental Validation}

\subsection{Experiments on Synthetic Datasets}

We evaluated our method using images synthesized from the MERL BRDF database [11]. For each material, we rendered four images of a sphere with light directions randomly sampled over the visible hemisphere. We transformed the known normals and light directions using a GBR transformation whose parameters are randomly sampled in the search space. These four images and the GBR-distorted normals and lighting directions are fed into our algorithm for test. We compared the recovered normal directions with ground truth and recorded the mean angular error.

For each material in the database, we repeated the above process 10 times with different light directions and GBR parameters. A plot of median error is shown in Figure 4 for all the BRDFs in the database. The results show that the proposed method is capable of recovering surface normals from the GBR ambiguity with high accuracy. Note that even some BRDFs are not strictly half-vector symmetric, the correct solutions still correspond to the global optimal of the objective function.

\subsection{Experiments on Real Object Datasets}

We have also evaluated our method on real data. Each dataset consists of a few images of an object with homoge- neous specular reflection. As a first step, color information was used to separate each image into a diffuse image and a specular image [16]. To handle shadows, the algorithm in [4] was used to fill in missing values in shadow regions. After pre-processing, we applied the technique of uncalibrated Lambertian photometric stereo to obtain normals and light directions up to an unknown GBR tranformation [26]. Finally, our coarse-to-fine optimization was performed using the specular images to fully recover surface normals.

To assess quality of the recovered normals, we compared our results with normals computed from calibrated photometric stereo. In the calibrated method, lighting information was known and the same set of diffuse images were used. As shown on the left of Figure 6, our algorithm successfully recovered normals of the apple surface and the recovered BRDF slice shows the expected low-rank structure, which is also observed in the result of calibrated photometric stereo. Figure 7 shows results on other datasets.

Comparison of our method with several other algorithms is shown in Table 1 . Those $[1,17,8]$ utilizing only albedo information were run on diffuse images. As can be seen, our method achieves a similar performance as the diffuse maxima method [8] and outperforms the other methods. Please notice that our method and [8] exploit completely different sources of information. While [8] is purely based on diffuse images and owns its robustness to a robust estimator, our method exploits information in specular reflection and its accuracy comes from a robust global structure. These two methods can reinforce each other and better performance can be expected by fusing them.

It is worth noting that in most examples, the BRDF slice recovered by our method shows an even better 'lowrank' pattern than that of the slice from calibrated photometric stereo. This difference suggests inaccuracy of recorded light directions. We carefully conducted another experiment to further validate our method. By painting a sphere with green paint whose BRDF is known, we prepared a dataset with known ground truths for both normals 


\begin{tabular}{|c||c|c|c|c|}
\hline Method & Duck & Apple & Pear & Pear2 \\
\hline$[1]$ & $7.5(5.4)$ & $9.0(3.9)$ & $9.7(3.6)$ & $23.8(9.4)$ \\
\hline$[17]$ & $6.6(5.5)$ & $8.9(3.6)$ & $24.9(8.3)$ & $23.7(10.3)$ \\
\hline$[6]$ & $7.7(4.4)$ & $8.7(3.9)$ & $4.6(2.4)$ & $13.8(5.3)$ \\
\hline$[21]$ & $7.3(9.4)$ & $9.8(16.2)$ & N/A & N/A \\
\hline$[8]$ & $7.4(4.8)$ & $\mathbf{7 . 0}(2.9)$ & $7.3(2.7)$ & $\mathbf{9 . 2}(4.9)$ \\
\hline Ours & $\mathbf{5 . 7}(4.5)$ & $7.8(3.1)$ & $\mathbf{4 . 4}(2.4)$ & $11.7(4.9)$ \\
\hline
\end{tabular}

Table 1. Mean and standard deviation(in brackets) of angular error(deg) by different methods on four datasets. Normals obtained from calibrated photometric stereo are regarded as ground truth.

\begin{tabular}{|c||c|c|}
\hline Method & Normal error & BRDF error \\
\hline Calibrated & $4.03(2.87)$ & 0.091 \\
\hline Ours & $3.95(1.87)$ & 0.071 \\
\hline
\end{tabular}

Table 2. The first column shows mean and standard deviation(in brackets) of angular error(deg) of normals by different methods on the Sphere dataset; the second column shows the root-meansquare error of recovered BRDF slices.

and the BRDF. After separating six images of the sphere under directional lighting into diffuse and specular components, both the calibrated method and our method were run respectively. Since the BRDF of the sphere is homogeneous across its surface, we used the original images instead of separated specular images in our algorithm to solve the GBR ambiguity. We then estimated the BRDF slice from each image based on normals(and light directions) recovered from both methods respectively. Recovered normals and BRDF slices were compared to ground truths. As can be seen from Figure 5 and Table 2, our method performed slightly better than the calibrated method in this case.

\section{Conclusion and Discussion}

In this paper, we have carefully examined the structure of a 2D BRDF slice estimated from a curved surface under directional illumination. We have shown that if the BRDF is bivariate, which is implied by isotropy and half-vector symmetry, the estimated BRDF slice will have a special low-rank structure and this structure is generally destroyed by GBR-distorted normals and light directions. Based on this observation, we have formulated a simple algorithm to automatically calibrate photometric stereo by restoring the structure. Our approach is distinguished from several previous works in that it seeks to recover a global structure instead of relying on a few critical surface points. This holistic approach makes our algorithm robust and accurate.

Limitations As implied by the algorithm, a major limitation to our method is that it requires sufficent normal variation to work. In fact, while information at all pixels with different normals helps achieve robustness, it excludes our method from dealing with extreme cases where only a few different normals are observed in an image. A possible solution is to use special light configurations [28]. Alternatively, we might also fit parametric models to these limited obser-

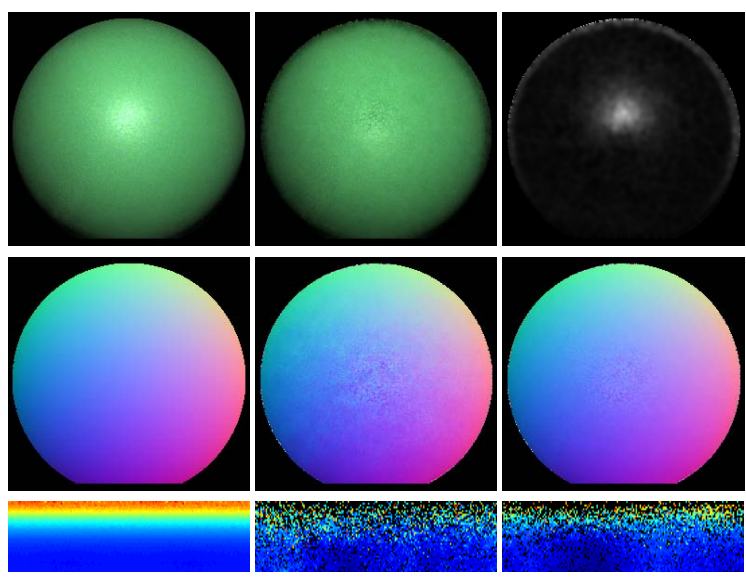

Figure 5. Results of the dataset Sphere. The first row shows the separation result of an image. The second row shows normals and the last row displays BRDF slices. From left to right are ground truth, results estimated from calibrated method, results produced by our method.

vations before analyzing the structure of the BRDF slice.

\section{Acknowledgements}

We thank the reviewers for their helpful suggestions. This project is partially supported by the AOARD and ONRG project R-263-000-A36-597.

\section{References}

[1] N. Alldrin, S. Mallick, and D. Kriegman. Resolving the generalized bas-relief ambiguity by entropy minimization. In Proc. CVPR, pages 1-7. IEEE, 2007. 2, 5, 6

[2] N. Alldrin, T. Zickler, and D. Kriegman. Photometric stere$\mathrm{o}$ with non-parametric and spatially-varying reflectance. In Proc. CVPR, pages 1-8. IEEE, 2008. 3

[3] P. Belhumeur, D. Kriegman, and A. Yuille. The basrelief ambiguity. International Journal of Computer Vision, 35(1):33-44, 1999. 1, 2

[4] M. Brand. Incremental singular value decomposition of uncertain data with missing values. In Proc. ECCV, pages $707-$ 720. Springer, 2002. 5

[5] M. Chandraker, F. Kahl, and D. Kriegman. Reflections on the generalized bas-relief ambiguity. In Proc. CVPR, volume 1, pages 788-795. IEEE, 2005. 2

[6] O. Drbohlav and M. Chaniler. Can two specular pixels calibrate photometric stereo? In Proc. ICCV, volume 2, pages 1850-1857. IEEE, 2005. 1, 2, 6

[7] O. Drbohlav and R. Šára. Specularities reduce ambiguity of uncalibrated photometric stereo. In Proc. ECCV, pages 644645. Springer, 2002. 1, 2

[8] P. Favaro and T. Papadhimitri. A closed-form solution to uncalibrated photometric stereo via diffuse maxima. In Proc. CVPR, pages 821-828. IEEE, 2012. 1, 2, 5, 6

[9] A. Georghiades. Incorporating the torrance and sparrow model of reflectance in uncalibrated photometric stereo. In Proc. ICCV, pages 816-823. IEEE, 2003. 2 

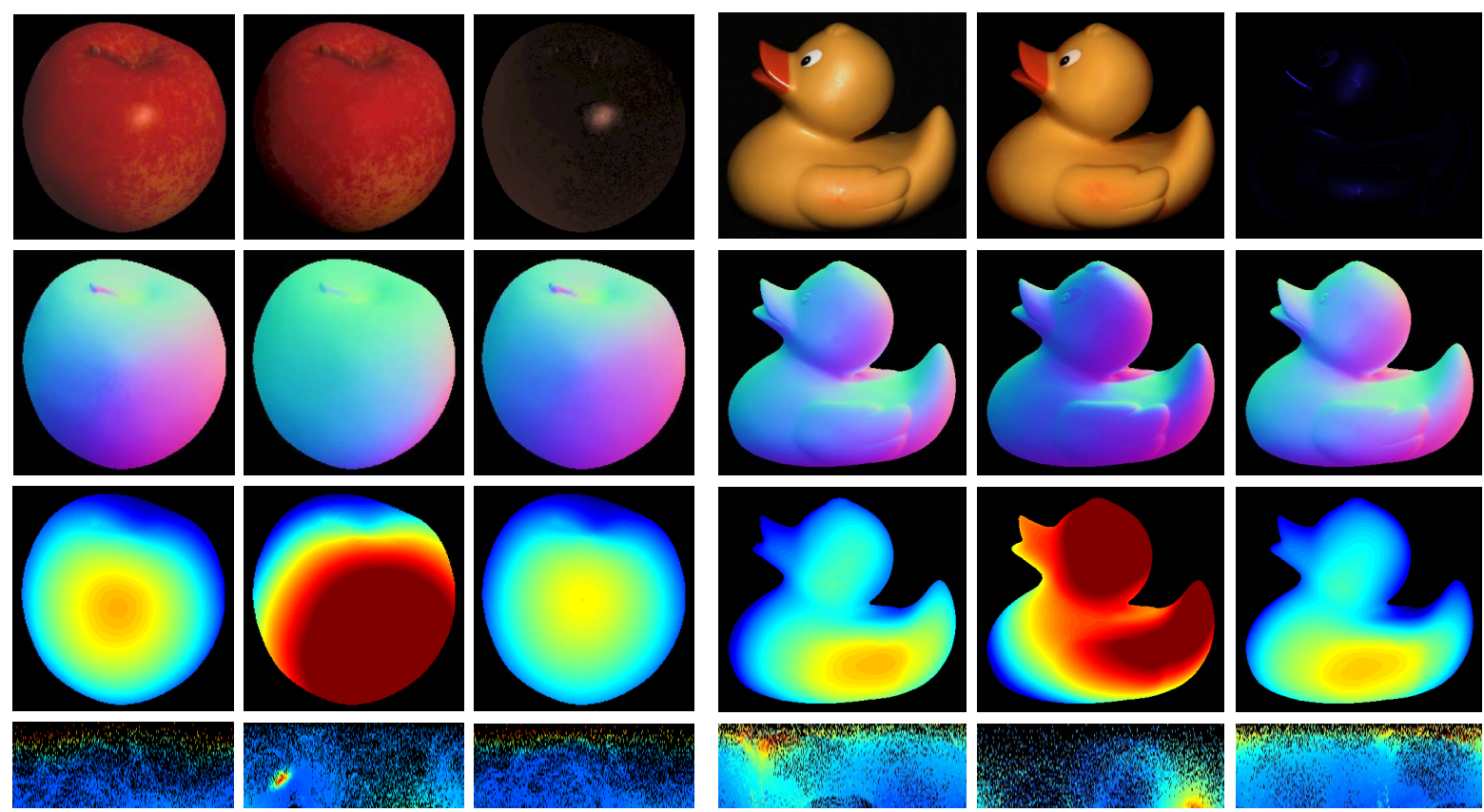

Figure 6. Results of the datasets Apple(left) and Duck (right). For each example, top row shows the input images, from left to right: an original image, separated diffuse image and specular image; Second row are normal maps encoded into colors $((R, G, B)=(\mathbf{n}+1) / 2)$, from left to right: calibrated photometric stereo, GBR distorted normals, our recovered normals; Third row shows the height map integrated from the normal field above it. The last row shows one of the recovered 2D BRDF slices using normals above it. Note that only rows $\theta_{h} \in\left[0^{\circ}, \ldots, 40^{\circ}\right]$ are shown.
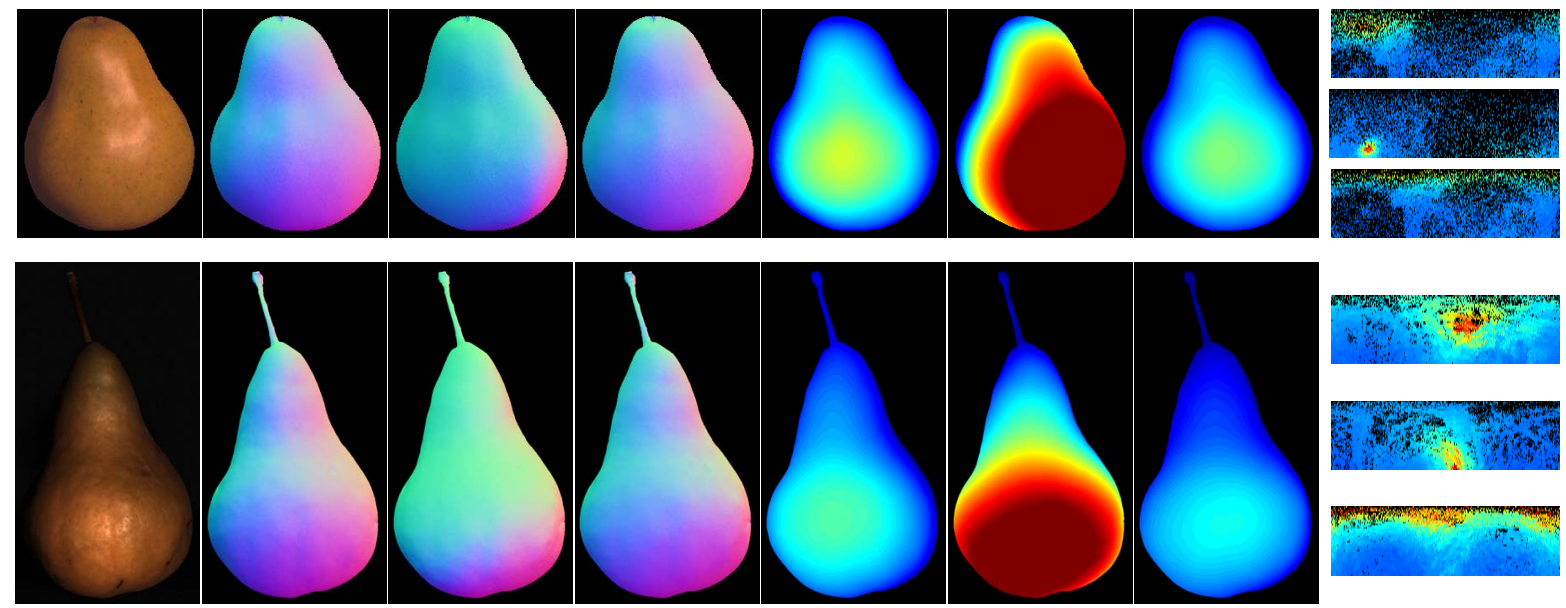

Figure 7. Results of datasets Pear(top) and Pear2(bottom). From left to right: an input image, normals from calibrated photometric stereo, GBR-distorted normals, our recovered normals, height map from calibrated photometric stereo, height map using GBR-distorted normals, our recovered height map, BRDF slices. In the last column, there are three BRDF slices for each dataset. From top to bottom, they are estimated using normals from calibrated photometric stero, GBR-distorted normals and our recovered normals respectively.

[10] H. Hayakawa. Photometric stereo under a light source with arbitrary motion. JOSA A, 11(11):3079-3089, 1994. 1, 2

[11] L. Mcmillan, A. Smith, and W. Matusik. A data-driven reflectance model. In Proc. of SIGGRAPH, 2003. 3, 5

[12] D. Nehab, S. Rusinkiewicz, J. Davis, and R. Ramamoorthi. Efficiently combining positions and normals for precise $3 \mathrm{~d}$ geometry. In ACM Transactions on Graphics (TOG), vol- ume 24, pages 536-543. ACM, 2005. 1

[13] T. Okatani and K. Deguchi. Optimal integration of photometric and geometric surface measurements using inaccurate reflectance/illumination knowledge. In Proc. CVPR, pages 254-261. IEEE, 2012. 1

[14] F. Romeiro, Y. Vasilyev, and T. Zickler. Passive reflectometry. In Proc. ECCV, pages 859-872. Springer, 2008. 3 
[15] S. Rusinkiewicz. A new change of variables for efficient brdf representation. In Eurographics Workshop on Rendering, pages 11-22, 1998. 1, 2, 3

[16] Y. Sato and K. Ikeuchi. Temporal-color space analysis of reflection. JOSA A, 11(11):2990-3002, 1994. 4, 5

[17] B. Shi, Y. Matsushita, Y. Wei, C. Xu, and P. Tan. Selfcalibrating photometric stereo. In Proc. CVPR, pages 11181125. IEEE, 2010. 2, 5, 6

[18] B. Shi, P. Tan, Y. Matsushita, and K. Ikeuchi. A biquadratic reflectance model for radiometric image analysis. In Proc. CVPR, pages 230-237. IEEE, 2012. 3

[19] M. Stark, J. Arvo, and B. Smits. Barycentric parameterizations for isotropic brdfs. IEEE Trans. on Visualization and Computer Graphics, 11(2):126-138, 2005. 1, 3

[20] P. Tan, S. Mallick, L. Quan, D. Kriegman, and T. Zickler. Isotropy, reciprocity and the generalized bas-relief ambiguity. In Proc. CVPR, pages 1-8. IEEE, 2007. 1, 2

[21] P. Tan, L. Quan, and T. Zickler. The geometry of reflectance symmetries. IEEE Trans. Pattern Anal. Mach. Intell., 33(12):2506-2520, 2011. 2, 6

[22] P. Tan and T. Zickler. A projective framework for radiometric image analysis. In Proc. CVPR, pages 2977-2984. IEEE, 2009. 1,2

[23] R. Tan and K. Ikeuchi. Separating reflection components of textured surfaces using a single image. Digitally Archiving Cultural Objects, pages 353-384, 2008. 4

[24] K. Torrance and E. Sparrow. Theory for off-specular reflection from roughened surfaces. JOSA, 57(9):1105-1112, 1967. 2

[25] R. Woodham. Photometric method for determining surface orientation from multiple images. Optical engineering, 19(1):191139-191139, 1980. 1, 2

[26] A. Yuille and D. Snow. Shape and albedo from multiple images using integrability. In Proc. CVPR, pages 158-164. IEEE, 1997. 1, 2, 4, 5

[27] Z. Zhang, X. Liang, A. Ganesh, and Y. Ma. Tilt: transform invariant low-rank textures. In Proc. ACCV, pages 314-328. Springer, 2011. 4

[28] Z. Zhou and P. Tan. Ring-light photometric stereo. In Proc. ECCV, pages 265-279. Springer, 2010. 2, 6

\section{A. Proof}

Proof. Consider the problem on the projective plane. Apart from a BRDF value, each point is also associated with a shading value $\mathbf{n}^{\top} \mathbf{s}$ and an intensity value $I$ which is the BRDF multiplied by the shading. For a bivariate BRDF, the BRDF map consists of a set of ellipses around $\mathbf{h}$ with a symmetry axis $v s$. It is clear the shading map is also symmetric about $v s$. So the intensity map is symmetric about $v s$ and its maximum $\mathbf{m}$ must lie on $v s$.

When the GBR ambiguity is incurred, the transformed light direction $\hat{\mathbf{s}}$ will keep lying on the line $v s$ based on equation 3.3 , and so is $\hat{\mathbf{h}}$. However, the intensity maximum will experience a translation $(\mu, \nu)$ and a scaling $\lambda$. If $(\mu, \nu)$ is not parallel to $v s$, the intensity maximum will shift away

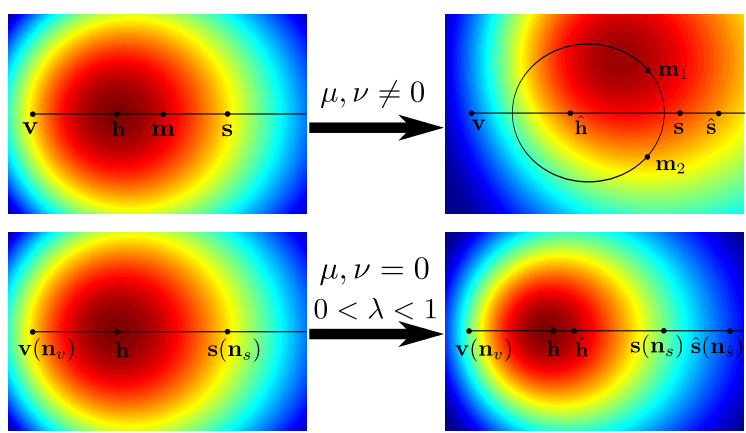

Figure 8. Projective plane with BRDF values. Top: BRDF transformed by a general GBR; bottom: BRDF transformed by GBR with $\mu, \nu=0,0<\lambda<1$.

from $v s$ to $\mathbf{m}_{1}$. Now we consider the point $\mathbf{m}_{2}$, which lies on the other side of $v s$ and is the mirror point of $\mathbf{m}_{1}$ across $v s$. Its intensity is smaller than that of $\mathbf{m}_{1}$ and their transformed shading values are equal: $\mathbf{m}_{1}^{\top} \hat{\mathbf{s}}=\mathbf{m}_{2}^{\top} \hat{\mathbf{s}}$. Thus, their transformed BRDF values are different. On the other hand, these two points have the same transformed half angle and thus they belong to the same row of the GBR distorted 2D BRDF slice. So the low-rank structure is broken.

Even if $(\mu, \nu)$ is parallel to $v s$, the GBR transformation will still break the low-rank structure of the BRDF slice from another image whose lighting direction is not coplanar with $\mathbf{v}$ and $\mathbf{s}$.

In the following, we consider the case $\mu, \nu=0$. We assume $\lambda>0$ by ignoring the concave/convex ambiguity. We will prove by contradiction for the case $\lambda<1$. The case of $\lambda>1$ can be proved similarly. Assume the low-rank structure still hold in the GBR-transformed BRDF slice.

Since the normals $\mathbf{n}_{v}=\mathbf{v}$ and $\mathbf{n}_{s}=\mathbf{s}$ have the same half angle: $\theta_{h}=\theta_{d}$, they should have the same BRDF value

$$
\frac{I\left(\mathbf{n}_{v}\right)}{\cos \left(2 \theta_{d}\right)}=\frac{I\left(\mathbf{n}_{s}\right)}{\cos (0)} \text {. }
$$

A similar relation holds for the GBR transformed case

$$
\frac{\hat{I}\left(\mathbf{n}_{v}\right)}{\cos \left(2 \hat{\theta}_{d}\right)}=\frac{\hat{I}\left(\mathbf{n}_{\hat{s}}\right)}{\cos (0)}
$$

where $\hat{I}$ is the transformed intensity map, $\mathbf{n}_{\hat{s}}=\hat{\mathbf{s}}$, and $\hat{\theta}_{d}$ is the transformed half angle for $\mathbf{n}_{v}$ and $\mathbf{n}_{\hat{s}}$. As shown in Section 3.3, $\hat{I}$ is the result of shrinking $I$ toward $\mathbf{v}$ and $\hat{\mathbf{s}}$ is further from $\mathbf{v}$ than $\mathbf{s}$, we have $I\left(\mathbf{n}_{v}\right)=\hat{I}\left(\mathbf{n}_{v}\right)$ and $\theta_{d}<\hat{\theta}_{d}$. Combining this with Equation (A.1,A.2), it can be derived that the relation $\hat{I}\left(\mathbf{n}_{\hat{s}}\right)>I\left(\mathbf{n}_{s}\right)$ should hold. On the other hand, $\mathbf{n}_{\hat{s}}$ is transformed from $\mathbf{n}_{x}$, which lies even further from $\mathbf{v}$ : $\hat{I}\left(\mathbf{n}_{\hat{s}}\right)=I\left(\mathbf{n}_{x}\right)$. For most realworld BRDFs, Intensity decreases as the normal moves away from the fixed viewing direction $\mathbf{v}$ and lighting direction s: $I\left(\mathbf{n}_{x}\right)<I\left(\mathbf{n}_{s}\right)$. thus, $\hat{I}\left(\mathbf{n}_{\hat{s}}\right)<I\left(\mathbf{n}_{s}\right)$. Contradiction. 